\title{
Stimulated Electronic X-Ray Raman Scattering
}

\author{
Clemens Weninger, ${ }^{1,2}$ Michael Purvis, ${ }^{3}$ Duncan Ryan, ${ }^{3}$ Richard A. London, ${ }^{4}$ John D. Bozek,${ }^{5}$ Christoph Bostedt, ${ }^{5}$ \\ Alexander Graf, ${ }^{4}$ Gregory Brown, ${ }^{4}$ Jorge J. Rocca, ${ }^{3}$ and Nina Rohringer ${ }^{1,2, *}$ \\ ${ }^{1}$ Max Planck Institute for the Physics of Complex Systems, 01187 Dresden, Germany \\ ${ }^{2}$ Center for Free-Electron Laser Science, 22761 Hamburg, Germany \\ ${ }^{3}$ Colorado Sate University, Fort Collins, Colorado 80523, USA \\ ${ }^{4}$ Lawrence Livermore National Laboratory, Livermore, California 94551, USA \\ ${ }^{5}$ LCLS, SLAC National Accelerator Laboratory, Menlo Park, California 94025, USA
}

(Received 16 June 2013; published 5 December 2013)

\begin{abstract}
We demonstrate strong stimulated inelastic $\mathrm{x}$-ray scattering by resonantly exciting a dense gas target of neon with femtosecond, high-intensity x-ray pulses from an x-ray free-electron laser (XFEL). A small number of lower energy XFEL seed photons drive an avalanche of stimulated resonant inelastic $\mathrm{x}$-ray scattering processes that amplify the Raman scattering signal by several orders of magnitude until it reaches saturation. Despite the large overall spectral width, the internal spiky structure of the XFEL spectrum determines the energy resolution of the scattering process in a statistical sense. This is demonstrated by observing a stochastic line shift of the inelastically scattered x-ray radiation. In conjunction with statistical methods, XFELs can be used for stimulated resonant inelastic x-ray scattering, with spectral resolution smaller than the natural width of the core-excited, intermediate state.
\end{abstract}

DOI: 10.1103/PhysRevLett.111.233902

Nonlinear spectroscopies [1,2] such as multidimensional [3] and fs stimulated Raman spectroscopy [4] have become standard techniques in the field of chemical reaction dynamics, combining high energy with high temporal resolution. X-ray free electron lasers (XFELs) [5-7] with pulse durations in the fs range and unprecedented high intensities, hold the potential for transferring these techniques to the $\mathrm{x}$-ray domain, to ultimately study the coherent interplay of electronic and vibrational degrees of freedom with high temporal and spatial resolution [8-10]. The high penetration depth of $\mathrm{x}$ rays, combined with the element and chemical sensitivity of inelastic x-ray scattering [11-13] could open pathways to temporally resolve complex dynamical processes such as energy transfer in light harvesting complexes or reaction dynamics of catalytic processes [14,15]. However, the cross section for x-ray Raman scattering is small compared to that in the visible spectral domain. Therefore, even at XFELs, single shot measurements are challenging, especially in dilute samples in the gas or liquid phase.

This difficulty could be overcome by stimulating the Raman scattering process to produce a strong coherent amplification of the signal. We present the first demonstration of stimulated resonant electronic x-ray Raman scattering (SRXRS) in an atomic gas. Resonant X-ray Raman scattering, also referred to as resonant inelastic $\mathrm{x}$-ray scattering [12], was discovered in 1974 [11] and since then has developed into a standard tool to study electronic and vibrational excitations in solids [16-18], liquids [19], gases [20], and on surfaces and interfaces [21,22]. Although several theoretical feasibility studies of SRXRS have been presented [23-26], no experiment has yet demonstrated this effect.

SRXRS would ideally be realized by a narrow-band, tuneable two-color $\mathrm{x}$-ray source: one frequency to excite an
PACS numbers: 42.55.Vc, 32.30.Rj, 42.65.Dr, 78.70.Ck

electronic inner-shell transition and the other, lower frequency to dump an electron into the short-lived core hole. Although the production of narrow-band $\mathrm{x}$ rays has been realized [27,28], and schemes for two-color self seeded sources have been proposed [29], there are currently no tunable, two-color, coherent sources available to perform such an experiment. Instead of this ideal, presently unrealizable approach, we report the demonstration of SRXRS in neon with a self-amplified spontaneous emission (SASE) XFEL [30]. SASE XFELs provide X-ray pulses of a noiselike spectrum with a bandwidth of several eV. This Letter demonstrates that, despite this broad width, the incoherent, spectrally structured SASE pulses give high energy resolution of the scattering process. Compared to the spontaneous RXRS process, a signal enhancement of 7-9 orders of magnitude was achieved. SRXRS is positioned to become a powerful method for pump-probe studies at XFELs.

In the experiment, pulses from the LCLS XFEL [6] of $\approx 40$ fs duration were focused into a gas cell filled with neon at a pressure of $\approx 500$ Torr [Fig. 1(a)]. The on-axis $\mathrm{x}$-ray radiation was analyzed with a flat-field grazing incidence spectrograph with an $\mathrm{x}$-ray CCD detector. The XFEL photon energy was varied across the $K$ edge of neon $(870.2 \mathrm{eV})$. For photon energies below the $K$ edge, neutral atoms are core excited by resonant excitation of the $1 \mathrm{~s}$ shell (see level scheme in Fig. 2). Due to the high intensities of the focused XFEL beam $\left(\approx 10^{17} \mathrm{~W} / \mathrm{cm}^{2}\right)$, the resonant population transfer happens on a fs time scale, comparable to the lifetime of the core-excited states $(\approx 2.5 \mathrm{fs})$. Thereby, a sizeable, but transient population inversion between the $1 s$ and the valence shell is achieved. Despite the small branching ratio of radiative versus Auger decay of $\approx 1.7 \%$, a few seed photons at the $1 s-2 p$ emission 


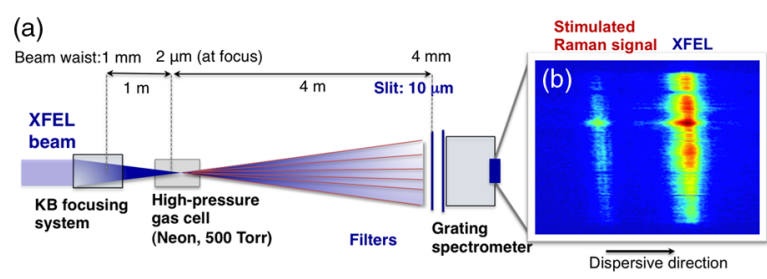

FIG. 1 (color online). (a) Experimental set up. The XFEL pulses are focused into a gas cell of $9.5 \mathrm{~mm}$ length filled with neon to spot sizes of $\approx 2 \mu \mathrm{m}$ using a Kirkpatrick-Baez (KB) focusing optics. A grating spectrometer of $\approx 1 \mathrm{eV}$ energy resolution is fielded downstream. (b) Typical raw spectrum.

frequency suffice to drive an avalanche of stimulated scattering events, which results in exponential amplification of the Raman signal. The process is similar to the recently demonstrated atomic inner-shell $\mathrm{x}$-ray laser [31], where population inversion is achieved by rapid $K$-shell photoionization. The geometry of this single-pass SRXRS amplifier is determined by the tight $(1-2 \mu \mathrm{m}$, determined by the focus spot size) and long ( $2 \mathrm{~mm}$, determined by the absorption length) longitudinally pumped gain medium. We measured the emission in the forward direction in a narrow cone of $\approx 1 \mathrm{mrad}$ divergence. The strongest $\mathrm{x}$-ray scattering channel involves the intermediary $1 s^{-1} 3 p{ }^{1} P_{1}$ level $(867.5 \mathrm{eV})$ and the final $2 p^{-1} 3 p^{1} S_{0}$ level, resulting in an emission at $849.3 \mathrm{eV}$. In a simple one-electron picture, the core-excited electron does not participate in this spectator decay. The process is stimulated by the spectral tail of the XFEL, providing $\sim 10^{4}-10^{5}$ seed photons/eV at $850 \mathrm{eV}$ for an incoming central photon energy of $867 \mathrm{eV}$ and $10^{12}$ photons per pulse. This big range is due to high irregularities of the XFEL spectrum. Irrespective of this variation of the seed flux, the effective stimulated scattering rate at the onset of the amplification process exceeds

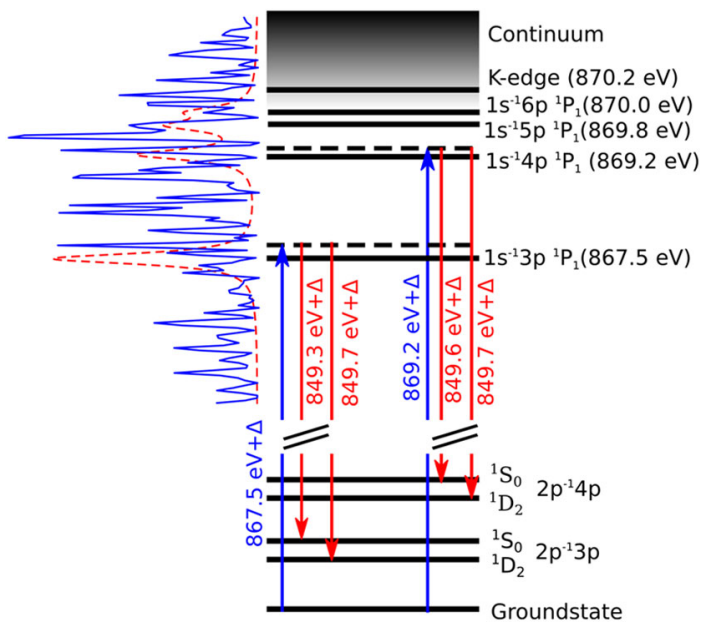

FIG. 2 (color online). Level scheme of neon. A simulated SASE pulse (blue solid line) overlaps with the Rydberg series of resonances and the continuum. The strongest spectral spike within the width of a resonance (red dashed line) drives the scattering. The energies and transition dipoles were calculated by the Hartree-Fock method [50]. the spontaneous scattering rate by $4-5$ orders of magnitude (see Supplemental Material [32]). It is, hence, dominantly the low-energy tail of the XFEL which serves as a seed of the SRXRS process. Our experimental setup did not allow measuring both the incoming and transmitted X-ray spectra at the same time. We measured a set of XFEL spectra without neon gas for reference. A cross correlation of the electron-beam energy of the accelerator with the measured central photon energy then allowed for a calibration of the XFEL photon energy (see Supplemental Material [32]).

Figure 3(a) shows the recorded emission spectra for XFEL photon energies $862<\omega<880 \mathrm{eV}$. The XFEL pulse follows the diagonal, showing an intensity drop due to absorption at the $1 s-3 p$ resonance and above the $K$ edge. The amplified line emission corresponds to the vertical structure at $\approx 850 \mathrm{eV}$, which was detected for $\omega$ as low as $864.9 \mathrm{eV}$. Because of the broad XFEL bandwidth (7 eV FWHM), covering the whole Rydberg series and part of the continuum, the SRXRS emission is not well separated from the $K$ - $\alpha$ emission above the edge; i.e., no pronounced variation of scattering intensity as a function of $\omega$ (for $\omega>867$ ) can be discerned.

A study of the line shape and position demonstrates that the emission for $\omega<870 \mathrm{eV}$ indeed stems from SRXRS: Figure 3(b) shows three single-shot spectra, each with a distinct emission line profile and peak position. The emission for $\omega=872 \mathrm{eV}$ has a narrower line width as compared to the spectra below the $K$ edge. Moreover, the peak position of the three lines is different. A systematic analysis of the measured line shape as a function of the XFEL photon energy is presented in Fig. 4(a). Since we want to compare line profiles, we omit spectra below $864.9 \mathrm{eV}$.

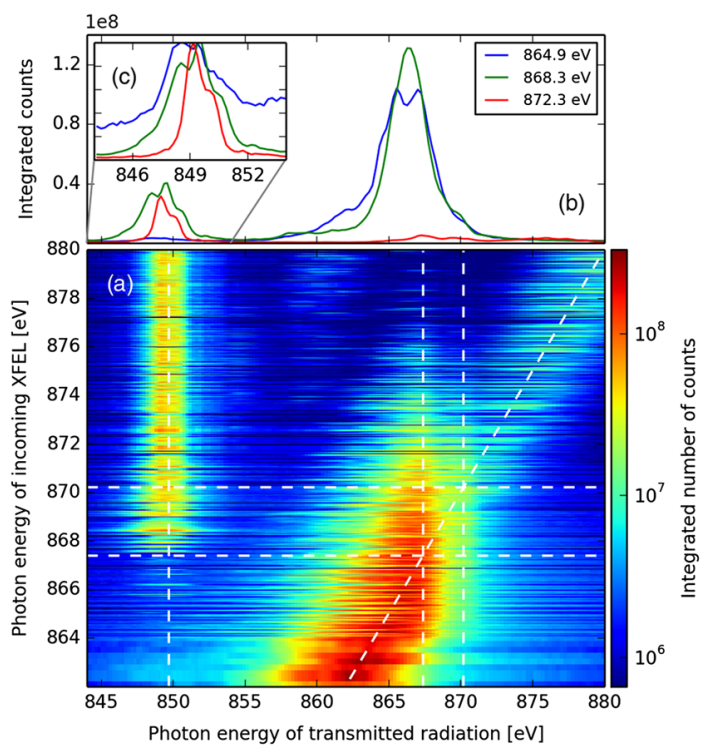

FIG. 3 (color online). (a) Measured single-shot spectra as a function of the incoming photon energy. 1028 single-shot spectra, with pulse energies ranging from 0.2 to $0.38 \mathrm{~mJ}$. The positions of the $1 s^{-1} 3 p$ transition $(\approx 867.5 \mathrm{eV})$, the $K$ edge $(\approx 870 \mathrm{eV})$, and the emission lines $(\approx 850 \mathrm{eV})$ are marked by dashed lines. (b) Three single-shot spectra. (c) Normalized emission lines. 


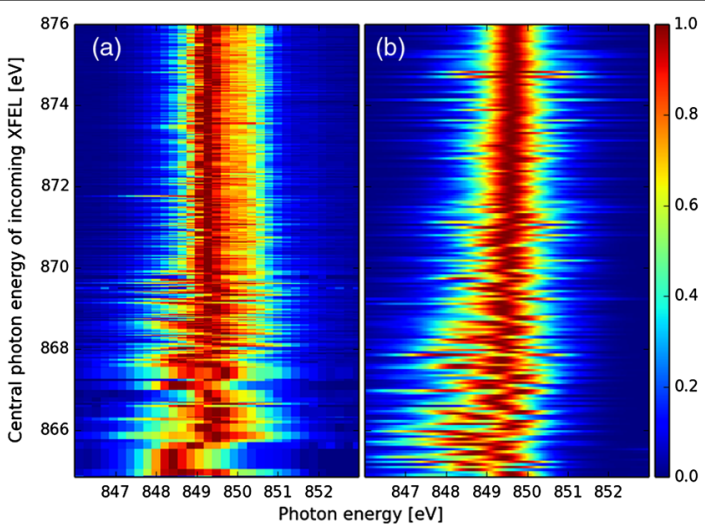

FIG. 4 (color online). (a) Measured emission-line profile as a function of the XFEL photon energy (876 single-shot spectra, of which 145 spectra are for energies below $870.2 \mathrm{eV}$ ). The spectra are normalized to their intensity maximum. Shown are only spectra of clearly isolated emission lines, with a peak at least a factor of 3 above background. Most of the lines have a signalto-noise ratio between 10 and 100. (b) Simulated emission spectra. The spectra were convolved with a Gaussian of a $1 \mathrm{eV}$ width to match the experimental resolution. We assumed $2 \times 10^{12}$ photons per pulse, a focal radius of $2 \mu \mathrm{m}$, an interaction length of $1 \mathrm{~cm}$ and $1.6 \times 10^{19}$ atoms $/ \mathrm{cm}^{3}$.

For this energy region, the XFEL tail substantially extends in the emission region and no isolated line emission could be detected. Figure 4(a) clearly shows a change of the line profile as a function of incoming photon energy: Whereas for $\omega>870 \mathrm{eV}$ the peak position $(849.2 \pm 0.1 \mathrm{eV})$ and the line shape are very reproducible from shot to shot, for $\omega<870 \mathrm{eV}$ the lines show a stochastic shift of $\pm 0.4 \mathrm{eV}$, broadening and sometimes a multipeak structure. These features can be linked to stochastic detuning of the multiple spectral spikes of the XFEL from resonance. To explain this effect, let us first consider monochromatic incoming $\mathrm{x}$ rays. Tuning the photon energy across an isolated resonance of energy $E_{2}-E_{1}$ results in emission of an inelastic scattered photon of energy $E_{2}-E_{3}+\Delta$ where $E_{2}-E_{3}$ denotes the energy difference between the intermediate and final state and $\Delta$ is the detuning from the resonance. The scattered light follows the linear energy dispersion of the resonance scattering process [11,33,34]. Now consider actual SASE pulses, which have a spiky spectrum within a broad envelope [35], as shown in Fig. 2. The average width of a spectral spike-the spectral coherence-is determined by the inverse pulse duration. In our case, this is $\approx 0.1 \mathrm{eV}$, narrower than the resonance width of $0.25 \mathrm{eV}$. Therefore, each spectral spike can be seen as a monochromatic x-ray pulse of a specific detuning, resulting in detuning of the emission energy. The process is dominated by one or a few of such spikes for each shot. Since the positions of the SASE spikes vary from pulse to pulse, this results in a stochastic line shift of the SRXRS signal. The linear dispersion of resonance scattering follows from the Kramers Heisenberg equation [12] which implies energy conservation only between the initial and final state. Thus, the intermediate, core-excited state does not have to be on the energy shell. This is in stark contrast to photoionization followed by fluorescence (for $\omega>870 \mathrm{eV}$ ). Here, energy conservation is enforced in both steps separately and the emission is always at fixed photon energy and has a Lorentzian line profile, irrespective of the bandwidth and central photon energy of the pump source. Pumping with a SASE XFEL, therefore, results in stable line position and line profile when tuned above the $K$ edge, and emission of variable line shape and stochastic line shifts when pumping below the $K$ edge, which results in resonant Raman scattering.

To support this interpretation, we have simulated SRXRS spectra by a theory, treating the atomic and ionic system self-consistently coupled to the propagation and amplification of the radiation. A theory summary is given in the Supplemental Material [32] and a detailed derivation and analysis of our theory was recently published [36]. To describe both the resonant contributions and the coupling to the continuum we need two density matrices: The density matrix of the neutral atom $\rho$, treating the resonant coupling of the ground, the core-excited intermediate $\left(1 s^{-1} n p^{1} P_{1} ; n=3, \ldots, 6\right)$, and final valence excited states $\left(2 p^{-1} n p^{1} S_{0}\right.$ and $\left.2 p^{-1} n p^{1} D_{2}\right)$. Because of the broad bandwidth of the XFEL, which are numerically simulated by Gaussian noise [37] of a bandwidth of $7 \mathrm{eV}$ and a pulse duration of $40 \mathrm{fs}$ (FWHM), the neutral atom can be core ionized. This introduces loss terms to the density matrix $\rho$ [24], but populates the reduced ionic density matrix $\rho^{1+}$ of the core-ionized state $\left(1 s^{-12} S_{1 / 2}\right)$, which decays to the ionic final states $\left(2 p^{-12} P_{1 / 2}\right.$ and $\left.2 p^{-12} P_{3 / 2}\right)$. The master equations determining the time evolution of $\rho$ and $\rho^{1+}$ can be derived by an open quantum system approach, starting from a configuration interaction singles approach $[36,38]$ and are self-consistently coupled to a 1D Maxwell equation $[39,40]$. In contrast to the previous theoretical treatment of SRXRS [24], where an effective SRXRS cross section based on second order perturbation theory was introduced, we treat coupling to the intermediate state explicitly. Our theory is valid to all orders in the photon interaction. Figure 4(b) shows calculated single-shot emission spectra as a function of the incoming photon energy. In agreement with the experiment, the emitted line shows stochastic detuning for $\omega<870 \mathrm{eV}$ and evolves into a reproducible form for $\omega>870 \mathrm{eV}$. Although the $1 s^{-1} 3 p^{1} P_{1}$ to $2 p^{-1} 3 p^{1} S_{0}$ transition has the strongest oscillator strength, contributions from other resonances are present. The competition of different SASE spikes in a single pulse results in a complex multipeak emission spectrum [36].

To demonstrate the exponential signal amplification, we studied the SRXRS signal as a function of the incoming pulse energy. Figure 5 shows experimental results compared to theory. We suppose a beam-line transmission of $18 \%$. Varying the pulse energy from 0.08 to $0.35 \mathrm{~mJ}$ results in a signal increase of almost 4 orders of magnitude. For $\omega>870 \mathrm{eV}$ the line emission is dominated by the 


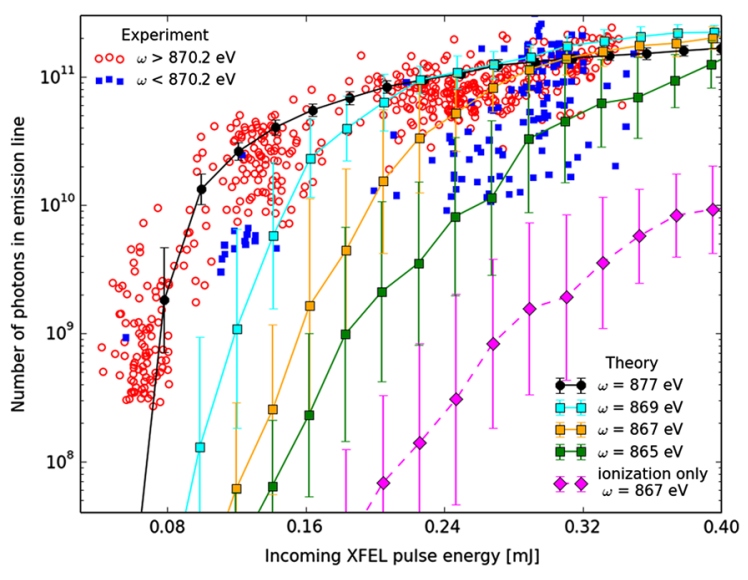

FIG. 5 (color online). Pump-energy dependence of the SRXRS signal: measured number of photons in the emission line as a function of the incoming pulse energy for $\omega>870 \mathrm{eV}$ (average $\bar{\omega}=876 \mathrm{eV})$ and $\omega<870 \mathrm{eV}(\bar{\omega}=868 \mathrm{eV})$ along with the theory results for $\omega=877,869,867$, and $865 \mathrm{eV}$. Each data point of the theory results corresponds to the geometric mean and standard deviation of 50 SASE pulses.

photoionization pumped x-ray laser and we clearly see exponential gain and saturation, in good quantitative agreement with theory. Despite the relatively low number of 145 data points for $\omega<870$, an exponential dependence of the SRXRS signal on the incoming pulse energy is observed. In contrast to the photoionization $x$-ray laser, the SRXRS signal shows a higher pulse-to-pulse fluctuation and is overall weaker, in agreement with theory. Although the coupling at resonance is enhanced, only $\sim 1 \%-10 \%$ of the XFEL photons overlap with the resonances. The SRXRS signal yield depends strongly on the incoming photon energy, as can be seen by our theory results in Fig. 5 . Despite the broad bandwidth of the incoming radiation, the efficiency of SRXRS drops considerably for central energies detuned below the $1 s-3 p$ resonance (compare theory for $\omega=865$ and $867 \mathrm{eV}$ ). Although the number of seed photons increases for decreasing photon energy, the reduction in the stimulated Raman gain cross section due to detuning results in lower amplification yields. Theoretical calculations for $\omega=862 \mathrm{eV}$ (see Supplemental Material [32]) show that no isolated line emission can be expected to rise above the background of the extended XFEL tail. The lack of isolated emission lines for $\omega<864 \mathrm{eV}$ [compare Fig. 3(a)] is, therefore, in agreement with theory. At $\omega=$ $869 \mathrm{eV}$ coupling to the continuum becomes important, resulting in higher emission yields. Although the experimental points are within the standard deviation of the theoretical ensemble averages, the theory slightly underestimates the experimental SRXRS yield at low pulse energies. Reasons for the discrepancy are strong irregularities of the XFEL emission, such as shot-to-shot variations of the bandwidth, pulse duration, spectral and temporal envelope, and sporadic spectral side bands to the lower energy side of the central photon energy. All of these changes were not included in our modeling. Whereas the total signal strength is less sensitive to those changes in the saturated regime, stronger dependence is expected in the exponential gain region. Considering that there are no free parameters in our model, the overall agreement between experiment and theory is remarkably good. To further support our claim that the signal for $\omega<870 \mathrm{eV}$ stems from SRXRS, we show numerical results for the resonance condition $\omega=867 \mathrm{eV}$, omitting the resonance contribution, i.e., only treating ionization-induced $\mathrm{x}$-ray emission. The signal lies 1-2 orders of magnitude lower than the detected value. The process of SRXRS is, therefore, dominating the emission feature for $\omega<870 \mathrm{eV}$ by far, and is crucial for explaining the high signal yield. It is noteworthy that spontaneous RXRS in the experimental geometry would yield $\sim 100$ photons on the detector, compared to the measured number of $10^{9}-10^{11}$.

We have presented strong experimental evidence for the observation of stimulated resonant electronic x-ray Raman scattering in neon gas. Compared to the spontaneous process, a signal enhancement up to a factor of $e^{16}-e^{21}$ was recorded, reaching saturated amplification. In contrast to amplified emission following photoionization, resulting in emission lines of reproducible shape and position, the resonant excitation with SASE pulses is characterized by a stochastic energy shift and a varying line shape. The spectral coherence of the XFEL ultimately defines the spectral resolution in a resonance scattering process, in a statistical sense [36]. Statistical methods, like covariance mapping [41] will be indispensable tools for analyzing the spectra and obtaining high-resolution 2D SRXRS maps [36]. Such analysis requires the recording of a large ensemble of single-shot spectra, which was beyond the scope of this experiment but is realistically achievable with the repetition rates at present-day XFELs. SRXRS and amplification by stimulated emission can be directly extended to molecular gases [42-44]. Indication of stimulated emission at around $90 \mathrm{eV}$ following photoionization of crystalline Silicon was recently reported [45], with a stimulated emission level of roughly twice the intensity of spontaneous emission. Aspects of this work, such as the build up of gain, the evolution of the emission spectrum as a function of pump-pulse energy, and the limits for saturation of the amplification process in transversely pumped solids, however, require further studies. Optimized experimental geometries, similar to traveling-wave excitations of transient solid-target soft x-ray lasers [46] need to be developed, so that directional, coherent signal amplification and SRXRS can be exploited in solids for spectroscopic purposes. The most general experimental geometry for SRXRS will not be scattering in forward direction, but will involve several x-ray beams of different angles of incidence. An upcoming beam line [47] allowing such a general setup is under construction at the first seeded short-wavelength FEL [28] and will set the stage for momentum resolved stimulated x-ray Raman scattering and transient x-ray gratings. Other possible experimental geometries, such as heterodyne detection for stimulated x-ray Raman scattering have 
also been discussed [26]. The recent demonstration of a two-color x-ray SASE scheme [48] might enable a new class of pump-probe techniques at XFEL sources based on SRXRS [49]. Looking forward, SRXRS might enable time-domain spectroscopy and nonlinear techniques in the x-ray domain with broad applications for studying ultrafast chemical and materials dynamics.

Portions of this work were carried out at the Linac Coherent Light Source, a national user facility operated by Stanford University on behalf of the U.S. Department of Energy, Office of Basic Energy Science. Part of this work was performed under the auspices of the U.S. Department of Energy by Lawrence Livermore National Laboratory (Contract No. DE-AC52-07NA27344). Support for the work of M.P., D. R., and J. J. R. by the U.S. Department of Energy, Office of Science, Basic Energy Sciences AMOS Program is acknowledged. We thank J. Nilsen and Chul-Min Kim for discussions, J. Dunn for filters, J.-C. Castagna, M. L. Swiggers, M. Messerschmidt, C.-M. Tsai, and S. F. CarronMontero for their assistance with the experiment, and M. J. Bogan and H. Chapman for the loan of an X-ray CCD camera. We are indebted to the LCLS operating team for their excellent support during beam time.

*nina@pks.mpg.de

[1] S. Mukamel, Principles of Nonlinear Optical Spectroscopy (Oxford University Press, New York, 1999).

[2] P. Hamm, M. Lim, and R. M. Hochstrasser, J. Phys. Chem. B 102, 6123 (1998).

[3] G. S. Engel, T. R. Calhoun, E.L. Read, T.-K. Ahn, T. Mančal, Y.-C. Cheng, R.E. Blankenship, and G. R. Fleming, Nature (London) 446, 782 (2007).

[4] P. Kukura et al., Science 310, 1006 (2005).

[5] W. Ackermann et al., Nat. Photonics 1, 336 (2007).

[6] P. Emma et al., Nat. Photonics 4, 641 (2010).

[7] T. Ishikawa et al., Nat. Photonics 6, 540 (2012).

[8] S. Tanaka and S. Mukamel, Phys. Rev. Lett. 89, 043001 (2002).

[9] U. Harbola and S. Mukamel, Phys. Rev. B 79, 085108 (2009).

[10] D. Healion, H. Wang, and S. Mukamel, J. Chem. Phys. 134, 124101 (2011).

[11] C. J. Sparks, Phys. Rev. Lett. 33, 262 (1974).

[12] F. Gel'mukhanov and H. Agren, Phys. Rep. 312, 87 (1999).

[13] U. Bergmann, P. Glatzel, and S. P. Cramer, Microchem. J. 71, 221 (2002).

[14] R. Alonso-Mori et al., Proc. Natl. Acad. Sci. U.S.A. 109, 19103 (2012).

[15] J. Kern et al., Science 340, 491 (2013).

[16] A. Kotani and S. Shin, Rev. Mod. Phys. 73, 203 (2001).

[17] J.-P. Rueff and A. Shukla, Rev. Mod. Phys. 82, 847 (2010).

[18] L. J. P. Ament, M.van Veenendaal, T. P. Devereaux, J. P. Hill, and J. van den Brink, Rev. Mod. Phys. 83, 705 (2011).

[19] L. Weinhardt, M. Blum, O. Fuchs, A. Benkert, F. Meyer, M. Bär, J. D. Denlinger, W. Yang, F. Reinert, and C. Heske, J. Electron Spectrosc. Relat. Phenom. 188, 111 (2013).

[20] F. Hennies, A. Pietzsch, M. Berglund, A. Föhlisch, T. Schmitt, V. Strocov, H. O. Karlsson, J. Andersson, and J.-E. Rubensson, Phys. Rev. Lett. 104, 193002 (2010).
[21] M. Nyberg, J. Hasselstrom, O. Karis, N. Wassdahl, M. Weinelt, A. Nilsson, and L. G. M. Pettersson, J. Chem. Phys. 112, 5420 (2000).

[22] A. Nilsson and L. G. M. Pettersson, Surf. Sci. Rep. 55, 49 (2004).

[23] E. E. Fill, S. J. van Enk, J. Zhang, and P. Lambropoulos, Phys. Rev. A 54, 5374 (1996).

[24] E. Hudis, P. L. Shkolnikov, and A. E. Kaplan, J. Opt. Soc. Am. B 11, 1158 (1994).

[25] Y.-P. Sun, J.-C. Liu, C.-K. Wang, and F. Gelmukhanov, Phys. Rev. A 81, 013812 (2010).

[26] B. D. Patterson, SLAC Technical Note SLAC-TN-10-026, 2010.

[27] G. Geloni, V. Kocharyan, and E. Saldin, J. Mod. Opt. 58, 1391 (2011)

[28] J. Amann et al., Nat. Photonics 6, 693 (2012).

[29] Y. Ding, Z. Huang, and R. D. Ruth, Phys. Rev. ST Accel. Beams 13, 060703 (2010).

[30] R. Bonifacio, C. Pellegrini, and L. Narducci, Opt. Commun. 50, 373 (1984).

[31] N. Rohringer et al., Nature (London) 481, 488 (2012).

[32] See Supplemental Material at http://link.aps.org/ supplemental/10.1103/PhysRevLett.111.233902 for the estimate of the absolute signal strength, calibration of the XFEL central photon energy, a summary of the theoretical approach and a discussion of the role of the XFEL as seed for the inelastic scattering process.

[33] P. Eisenberger, P. M. Platzman, and H. Winick, Phys. Rev. Lett. 36, 623 (1976)

[34] M. Oura, Plasma Sci. Technol. 12, 353 (2010).

[35] E. Saldin, E. Schneidmiller, and M. Yurkov, Opt. Commun. 148, 383 (1998).

[36] C. Weninger and N. Rohringer, Phys. Rev. A 88, 053421 (2013).

[37] N. Rohringer and R. Santra, Phys. Rev. A 76, 033416 (2007).

[38] N. Rohringer and R. Santra, Phys. Rev. A 86, 043434 (2012).

[39] O. Larroche, D. Ros, A. Klisnick, A. Sureau, C. Moller, and H. Guennou, Phys. Rev. A 62, 043815 (2000).

[40] C. M. Kim, K. A. Janulewicz, and J. Lee, Phys. Rev. A 84, 013834 (2011).

[41] L. J. Frasinski, K. Codling, and P. A. Hatherly, Science 246, 1029 (1989).

[42] Q. Miao, J.-C. Liu, H. Agren, J.E. Rubensson, and F. Gelmukhanov, Phys. Rev. Lett. 109, 233905 (2012).

[43] V. Kimberg and N. Rohringer, Phys. Rev. Lett. 110, 043901 (2013).

[44] V. Kimberg, S. B. Zhang, and N. Rohringer, J. Phys. B 46, 164017 (2013).

[45] M. Beye, S. Schreck, F. Sorgenfrei, C. Trabant, N. Pontius, C. Schüßler-Langeheine, W. Wurth, and A. Föhlisch, Nature (London) 501, 191 (2013).

[46] J. Dunn, Y. Li, A. L. Osterheld, J. Nilsen, J. R. Hunter, and V. N. Shlyaptsev, Phys. Rev. Lett. 84, 4834 (2000).

[47] F. Bencivenga and C. Masciovecchio, Nucl. Instrum. Methods Phys. Res., Sect. A 606, 785 (2009).

[48] A. A. Lutman, R. Coffee, Y. Ding, Z. Huang, J. Krzywinski, T. Maxwell, M. Messerschmidt, and H.-D. Nuhn, Phys. Rev. Lett. 110, 134801 (2013).

[49] S. Mukamel, D. Healion, Y. Zhang, and J. D. Biggs, Annu. Rev. Phys. Chem. 64, 101 (2013).

[50] R. D. Cowan, The Theory of Atomic Structure and Spectra (University of California Press, Berkeley, 1981). 